doi: $10.13108 / 2021-13-2-170$

\title{
ON DISCRETIZATION OF DARBOUX INTEGRABLE SYSTEMS ADMITTING SECOND-ORDER INTEGRALS
}

\author{
K. ZHELTUKHIN, N. ZHELTUKHINA
}

\begin{abstract}
We consider a discretization problem for hyperbolic Darboux integrable systems. In particular, we discretize continuous systems admitting $x$ - and $y$-integrals of the first and second order. Such continuous systems were classified by Zhyber and Kostrigina. In the present paper, continuous systems are discretized with respect to one of continuous variables and the resulting semi-discrete system is required to be also Darboux integrable.

To obtain such a discretization, we take $x$ - or $y$-integrals of a given continuous system and look for a semi-discrete systems admitting the chosen integrals as $n$-integrals. This method was proposed by Habibullin. For all considered systems and corresponding sets of integrals we were able to find such semi-discrete systems. In general, the obtained semidiscrete systems are given in terms of solutions of some first order quasilinear differential systems. For all such first order quasilinear differential systems we find implicit solutions. New examples of semi-discrete Darboux integrable systems are obtained. Also for each of considered continuous systems we determine a corresponding semi-discrete system that gives the original system in the continuum limit.
\end{abstract}

Keywords: Darboux integrability, discretization.

Mathematics Subject Classification: 37K60

\section{INTRODUCTION}

In the present paper we study the problem of the discretization of integrable equations so that the integrability property is preserved. In particular, we consider hyperbolic systems

$$
p_{x y}^{i}=f^{i}\left(x, y, p, p_{x}, p_{y}\right) \quad i=1, \ldots, m,
$$

where $p=\left(p^{1}, \ldots, p^{m}\right), p_{x}=\left(p_{x}^{1}, \ldots, p_{x}^{m}\right)$ and $p_{y}=\left(p_{y}^{1}, \ldots, p_{y}^{m}\right)$.

For such hyperbolic systems it is convenient to use Darboux integrability [1]. The above system is said to be integrable if it admits $m$ functionally independent non-trivial $x$-integrals and $m$ functionally independent non-trivial $y$-integrals. A function $I\left(x, y, p, p_{y}, p_{y y}, \ldots\right)$ is called an $x$-integral of the system 1.1 if

$$
D_{x} I\left(x, y, p, p_{y}, p_{y y}, \ldots\right)=0 \quad \text { for all solutions of } 1.1 \text {, }
$$

where $D_{x}$ is the total derivative with respect to $x$. One can define $y$-integrals in a similar way. Darboux integrable systems are extensively studied, see [2]-[11] and a review paper [12].

The extension of the notion of Darboux integrability to discrete and semi-discrete Darboux integrable systems was developed by Habibullin and Pekcan [13], see also [14]. In recent years there is an interest in studying such systems, see [15]-[25]. A semi-discrete system

$$
q_{1 x}^{i}=f^{i}\left(x, n, q, q_{x}, q_{1}\right) \quad i=1, \ldots, m,
$$

K. Zheltukhin, N. Zheltukhina, On discretization of Darboux integrable systems admitting SECOND-ORDER INTEGRALS.

(C) K. Zheltukhin, N. Zheltukhina. 2021.

Submitted August 3, 2020. 
where $q=\left(q^{1}, \ldots, q^{m}\right), q_{x}=\left(q_{x}^{1}, \ldots, q_{x}^{m}\right)$ and $q_{1}=\left(q^{1}(x, n+1), \ldots, q^{m}(x, n+1)\right)$, is called Darboux integrable if it admits $m$ functionally independent non-trivial $x$-integrals and $m$ functionally independent non-trivial $n$-integrals. A function $J\left(x, n, q, q_{x}, q_{x x}, \ldots\right)$ is called an $n$-integral of the system 1.3 if

$$
D J\left(x, n, q, q_{x}, q_{x x}, \ldots\right)=J\left(x, n, q, q_{x}, q_{x x}, \ldots\right) \quad \text { for all solutions of } 1.3 \text {, }
$$

where $D$ is the shift operator, that is $D q=q_{1}$. Note that $D q_{k}=q_{k+1}, k=1,2,3, \ldots$. The $x$-integrals $I\left(x, n, q, q_{1}, q_{2}, \ldots\right)$ for system $(1.3)$ are defined in the same way as for continuous systems.

A hypothesis states that any continuous Darboux integrable system can be discretized with respect to one of the independent variables such that the resulting semi-discrete system is Darboux integrable and admits the set of $x$ - or $y$-integrals of the original system as $n$-integrals [26]. The results of our work support the above conjecture. We complete the discretization of continuous Darboux integrable equations derived by Zhiber and Kostrigina in [8]. In their paper, Zhiber and Kostrigina considered the classification problem for continuous Darboux integrable systems with two integrals of the first order and two integrals of the second order. They found all such systems together with their $x$ - and $y$-integrals. Following [8], we have two types of systems. The first system is

$$
\left\{\begin{array}{l}
u_{x y}=\frac{u_{x} u_{y}}{u+v}+\left(\frac{1}{u+v}+\frac{\alpha}{u+\alpha^{2} v}\right) u_{x} v_{y}, \\
v_{x y}=\frac{\alpha^{2} v_{x} v_{y}}{u+\alpha^{2} v}+\left(\frac{1}{\alpha(u+v)}+\frac{1}{u+\alpha^{2} v}\right) u_{x} v_{y},
\end{array}\right.
$$

with $\alpha$ being a nonzero constant. We mention that in the case $\alpha=1$, system (1.5) was discretized in [26]. For $\alpha \neq 1$, it possesses $y$-integrals

$$
\begin{aligned}
& I_{1}=\left(1+\frac{1}{\alpha}\right) v\left(\frac{u_{x}}{u+v}\right)^{1-\alpha}-v_{x}\left(\frac{u_{x}}{u+v}\right)^{-\alpha}, \\
& J_{1}=\frac{u_{x x}}{u_{x}}-\frac{(\alpha+1) u_{x}+\alpha v_{x}}{\alpha(u+v)},
\end{aligned}
$$

and the $x$-integrals have the same form in $u, u_{y}, u_{y y}$ and $v, v_{y}, v_{y y}$ variables.

The second system reads as

$$
\left\{\begin{array}{l}
u_{x y}=\frac{v u_{x} u_{y}}{u v+d}+\left(\frac{1}{u v+d}+\frac{1}{\alpha(u v+c)}\right) u u_{x} v_{y}, \\
v_{x y}=\frac{u v_{x} v_{y}}{u v+c}+\left(\frac{\alpha}{u v+d}+\frac{1}{u v+c}\right) v u_{x} v_{y},
\end{array}\right.
$$

where $\alpha, c$ and $d$ are nonzero constants. For $\alpha=-1$ it possesses $y$-integrals

$$
I_{2}=\frac{(d-c) v^{2} u_{x}^{2}}{2(u v+d)^{2}}-\frac{c u_{x} v_{x}}{u v+d}
$$

and

$$
J_{2}=\frac{u_{x x}}{u_{x}}+\frac{(d-c) v u_{x}-c u v_{x}}{c(u v+d)},
$$

where $c$ and $d$ are non-zero constants and the $x$-integrals have the same form in $u, u_{y}, u_{y y}$ and $v, v_{y}, v_{y y}$ variables.

For $\alpha \neq-1$ it possesses $y$-integrals

$$
I_{3}=\frac{u_{x}^{\beta} v_{x}}{(u v+d)^{\beta}}+\frac{\beta v^{2} u_{x}^{\beta+1}}{(u v+d)^{\beta+1}}
$$


and

$$
J_{3}=-\frac{u_{x x}}{u_{x}}+\frac{2 v u_{x}+u v_{x}}{u v+d}
$$

where $d$ and $\beta=-\alpha \neq 1$ are nonzero constants, and the $x$-integrals have the same form in $u, u_{y}, u_{y y}$ and $v, v_{y}, v_{y y}$ variables.

In order to discretize the systems (1.5) and (1.8), we employ a method introduced by Habibullin et. all [20], see also [24]-[26]. According to this approach, one takes the $x$ - or $y$-integrals of a system and looks for a semi-discrete system admitting such integrals as $n$-integrals. In general, one gets a set of semi-discrete systems admitting these $n$-integrals. For all sets of the $y$-integrals of systems 1.5 and (1.8) we obtain corresponding semi-discrete systems. Note that initially we allow the parameters $\alpha, c$ and $d$ in integrals (1.6), (1.7) and (1.9)-(1.12) to depend on $n$. It turns out that only $d$ may depend non-trivially on $n$ in one case. In all cases we are able to choose a semi-discrete system that gives the original system in the continuum limit. Also in examples, where we can write a semi-discrete system explicitly, we show that the system is Darboux integrable.

The following theorems are formulated for a hyperbolic type semi-discrete system

$$
\left\{\begin{array}{l}
u_{1 x}=f\left(x, n, u, v, u_{x}, v_{x}, u_{1}, v_{1}\right), \\
v_{1 x}=g\left(x, n, u, v, u_{x}, v_{x}, u_{1}, v_{1}\right),
\end{array}\right.
$$

where variables $u, v$ depend on a continuous variable $x \in \mathbb{R}$ and a discrete variable $n \in \mathbb{Z}$.

Theorem 1.1. Let $\alpha \neq 1$. System (1.13) admits $n$-integrals 1.6 and (1.7) if and only if it is of the form

$$
\left\{\begin{array}{l}
u_{1 x}=\frac{u_{1}+v_{1}}{u+v} \mathcal{D}_{1}^{\alpha^{-1}} u_{x} \\
v_{1 x}=\frac{\alpha+1}{\alpha} \frac{v_{1} \mathcal{D}_{1}^{\alpha^{-1}}-v \mathcal{D}_{1}}{u+v} u_{x}+\mathcal{D}_{1} v_{x}
\end{array}\right.
$$

The function $\mathcal{D}_{1}$ is equal to 1 or given implicitly by $H\left(n, K_{1}, L_{1}\right)=0$, where, for each $n$, the symbol $H$ denotes an arbitrary smooth function and

$$
\begin{aligned}
& K_{1}=\frac{\alpha v_{1} \mathcal{D}_{1}^{\alpha^{-1}}-\alpha v \mathcal{D}_{1}^{1+\alpha^{-1}}+\left(1-\mathcal{D}_{1}^{\alpha^{-1}}\right) u_{1}}{\left(\mathcal{D}_{1}^{\alpha^{-1}}-1\right)^{\alpha+1}}, \\
& L_{1}=\frac{\left(u_{1}-\mathcal{D}_{1}^{1+\alpha^{-1}} u\right) e^{\mathcal{D}_{1}^{\alpha^{-1}}}}{\mathcal{D}_{1}\left(\mathcal{D}_{1}^{\alpha^{-1}}-1\right)^{a}}+\frac{(-1)^{\alpha} e^{\mathcal{D}_{1}^{\alpha}}\left(\alpha v_{1} \mathcal{D}_{1}^{\alpha^{-1}}-\alpha v \mathcal{D}_{1}^{1+\alpha^{-1}}+\left(1-\mathcal{D}_{1}^{\alpha^{-1}}\right) u_{1}\right)}{\left(\mathcal{D}_{1}^{\alpha^{-1}}-1\right)^{\alpha+1}} .
\end{aligned}
$$

Let us construct some examples.

Example 1.1. In the case $\mathcal{D}_{1}=1$ system (1.14) becomes

$$
\left\{\begin{array}{l}
u_{1 x}=\frac{u_{1}+v_{1}}{u+v} u_{x} \\
v_{1 x}=\left(1+\frac{1}{\alpha}\right) \frac{v_{1}-v}{u+v} u_{x}+v_{x} .
\end{array}\right.
$$

This system is Darboux integrable. Indeed, it possesses two independent non-trivial n-integrals (1.6), 1.7) and two independent non-trivial $x$-integrals

$$
\mathcal{F}_{1}=\frac{v-v_{1}}{v_{1}-v_{2}} \quad \text { and } \quad \mathcal{F}_{2}=\frac{u_{1}-u}{\left(v_{1}-v\right)^{\frac{\alpha}{1+\alpha}}}-\alpha\left(v_{1}-v\right)^{\frac{1}{1+\alpha}} .
$$

The $x$-integrals can be found by considering the $x$-ring corresponding to the system.

Example 1.2. Letting $K_{1}=0$ and $\alpha=-1$, we get

$$
\mathcal{D}_{1}=\frac{u_{1}+v_{1}}{u_{1}+v}
$$


Using (1.14, we find the system

$$
\left\{\begin{array}{l}
u_{1 x}=\frac{u_{1}+v}{u+v} u_{x}, \\
v_{1 x}=\frac{u_{1}+v_{1}}{u_{1}+v} v_{x} .
\end{array}\right.
$$

This system is Darboux integrable. It possesses two independent non-trivial n-integrals (1.6), (1.7) and two independent non-trivial x-integrals

$$
\mathcal{F}_{1}=\frac{\left(u_{2}+v_{1}\right)\left(v-v_{1}\right)}{\left(u_{1}+v_{1}\right)\left(v_{1}-v_{2}\right)} \quad \text { and } \quad \mathcal{F}_{2}=\frac{\left(u-u_{1}\right)\left(u_{1}+v_{1}\right)}{\left(-u_{1}+u_{2}\right)\left(u_{1}+v\right)} .
$$

Example 1.3. Choosing $K_{1}=0$ and $\alpha=-\frac{1}{2}$, we get

$$
\mathcal{D}_{1}=\frac{4 u_{1}+2 v_{1}}{v+\sqrt{v^{2}+16 u_{1}^{2}+8 u_{1} v_{1}}} .
$$

By (1.14, we obtain the system

$$
\left\{\begin{aligned}
u_{1 x}= & \frac{u_{1}+v_{1}}{u+v}\left(\frac{v+\sqrt{v^{2}+16 u_{1}^{2}+8 u_{1} v_{1}}}{4 u_{1}+2 v_{1}}\right)^{2} u_{x}, \\
v_{1 x}= & -\left(\frac{v_{1}}{u+v}\left(\frac{v+\sqrt{v^{2}+16 u_{1}^{2}+8 u_{1} v_{1}}}{4 u_{1}+2 v_{1}}\right)^{2}\right. \\
& \left.-\frac{v\left(4 u_{1}+2 v_{1}\right)}{(u+v)\left(v+\sqrt{v^{2}+16 u_{1}^{2}+8 u_{1} v_{1}}\right)}\right) u_{x} \\
& +\frac{4 u_{1}+2 v_{1}}{v+\sqrt{v^{2}+16 u_{1}^{2}+8 u_{1} v_{1}}} v_{x} .
\end{aligned}\right.
$$

This system possesses two independent non-trivial n-integrals (1.6) and (1.7).

Example 1.4. Considering $L_{1}=0$ and $\alpha=-1 / 2$, we get

$$
\mathcal{D}_{1}=\frac{v_{1}+\sqrt{v_{1}^{2}+16 u^{2}+8 u v}}{2 v+4 u} .
$$

By (1.14) we then arrive at the system

$$
\left\{\begin{array}{c}
u_{1 x}=\frac{u_{1}+v_{1}}{u+v}\left(\frac{2 v+4 u}{v_{1}+\sqrt{v_{1}^{2}+16 u^{2}+8 u v}}\right)^{2} u_{x} \\
v_{1 x}=-\left(\frac{v_{1}}{u+v}\left(\frac{2 v+4 u}{v_{1}+\sqrt{v_{1}^{2}+16 u^{2}+8 u v}}\right)^{2}-\frac{v\left(v_{1}+\sqrt{v_{1}^{2}+16 u^{2}+8 u v}\right)}{(u+v)(2 v+4 u)}\right) u_{x} \\
+\frac{v_{1}+\sqrt{v_{1}^{2}+16 u^{2}+8 u v}}{2 v+4 u} v_{x} .
\end{array}\right.
$$

This system possesses two independent non-trivial n-integrals (1.6) and (1.7).

Remark 1.1. In both previous examples let us consider the corresponding $x$-rings. Let

$$
\begin{aligned}
& X=D_{x}, \quad Y_{1}=\frac{\partial}{\partial u_{x}}, \quad Y_{2}=\frac{\partial}{\partial v_{x}}, \\
& E_{1}=\left[Y_{1}, X\right], \quad E_{2}=\left[Y_{2}, X\right], \quad E_{3}=\left[E_{1}, E_{2}\right] .
\end{aligned}
$$


We observe that

$$
X=u_{x} E_{1}+v_{x} E_{2}+Y_{1}+Y_{2} .
$$

The following multiplication table

\begin{tabular}{c|c|c|c}
{$\left[E_{i}, E_{j}\right]$} & $E_{1}$ & $E_{2}$ & $E_{3}$ \\
\hline$E_{1}$ & 0 & $E_{3}$ & $-2(u+v)^{-1} E_{3}$ \\
\hline$E_{2}$ & $-E_{3}$ & 0 & $-2(u+v)^{-1} E_{3}$ \\
\hline$E_{3}$ & $2(u+v)^{-1} E_{3}$ & $2(u+v)^{-1} E_{3}$ & 0
\end{tabular}

shows that the $x$-rings are finite-dimensional. Therefore, systems (1.21) and (1.22) are Darboux integrable.

Remark 1.2. We consider the function $\mathcal{D}_{1}^{\alpha^{-1}}$ defined implicitly by

$$
H\left(K_{1}, L_{1}\right)=K_{1}=0,
$$

that is, by

$$
\alpha v_{1} \mathcal{D}_{1}^{\alpha^{-1}}-\alpha v \mathcal{D}_{1}^{1+\alpha^{-1}}+\left(1-\mathcal{D}_{1}^{\alpha^{-1}}\right) u_{1}=0
$$

and expand it into a series of the form

$$
\mathcal{D}_{1}^{\alpha^{-1}}\left(u_{1}, v, v_{1}\right)=\sum_{n=0}^{\infty} a_{n}\left(v_{1}-v\right)^{n},
$$

where the coefficients $a_{n}$ depend on variables $u_{1}$ and $v$ only. This yields

$$
\mathcal{D}_{1}^{\alpha^{-1}}\left(u_{1}, v, v_{1}\right)=1+\frac{\alpha}{u_{1}+\alpha^{2} v}\left(v_{1}-v\right)+\sum_{n=2}^{\infty} a_{n}\left(v_{1}-v\right)^{n}
$$

and

$$
\mathcal{D}_{1}\left(u_{1}, v, v_{1}\right)=1+\frac{\alpha^{2}}{u_{1}+\alpha^{2} v}\left(v_{1}-v\right)+\sum_{n=2}^{\infty} a_{n}\left(v_{1}-v\right)^{n} .
$$

Letting $u_{1}=u+\varepsilon u_{y}, v_{1}=v+\varepsilon v_{y}$ and passing to the limit as $\varepsilon \rightarrow 0$, one can see that system (1.14) becomes (1.5).

Theorem 1.2. System (1.13) admits n-integrals (1.9) and (1.10) if and only if it is of the form

$$
\left\{\begin{array}{l}
u_{1 x}=\frac{v\left(u_{1} v_{1}+d\right) \mathcal{D}_{2}}{v_{1}(u v+d)} u_{x}, \\
v_{1 x}=\frac{(d-c) v v_{1}\left(\mathcal{D}_{2}^{2}-1\right)}{2 c(u v+d) \mathcal{D}_{2}} u_{x}+\frac{v_{1}}{v \mathcal{D}_{2}} v_{x} .
\end{array}\right.
$$

The function $\mathcal{D}_{2}$ is defined implicitly by $H\left(n, K_{2}, L_{2}\right)=0$, where, for each $n$, the symbol $H$ denotes an arbitrary smooth function and

$$
K_{2}=\frac{v_{1}\left(\mathcal{D}_{2}-1\right) M^{-\frac{2 d}{c+d}}}{v \mathcal{D}_{2}}\left(-2 c d u_{1} v_{1}+u v \mathcal{D}_{2} M\right), \quad L_{2}=\frac{v \mathcal{D}_{2} M^{\frac{2 d}{c+d}}}{v_{1}}
$$

where

$$
M=2 c d+\frac{(c+d)\left(\mathcal{D}_{2}-1\right) u_{1} v_{1}}{\mathcal{D}_{2}}
$$


Example 1.5. Let $K_{2}=0$, then we get

$$
\mathcal{D}_{2}=\frac{(2 c d+(c+d) u v) u_{1} v_{1}}{\left(2 c d+(c+d) u_{1} v_{1}\right) u v} .
$$

Using (1.23), we obtain the system

$$
\left\{\begin{aligned}
u_{1 x}= & \frac{u_{1}\left(u_{1} v_{1}+d\right)(2 c d+(c+d) u v)}{u(u v+d)\left(2 c d+(c+d) u_{1} v_{1}\right)} u_{x} \\
v_{1 x}= & \frac{(d-c)}{2 c(u v+d)}\left(\frac{u_{1} v_{1}^{2}(2 c d+(c+d) u v)}{u\left(2 c d+(c+d) u_{1} v_{1}\right)}-\frac{u v^{2}\left(2 c d+(c+d) u_{1} v_{1}\right)}{u_{1}(2 c d+(c+d) u v)}\right) u_{x} \\
& +\frac{u\left(2 c d+(c+d) u_{1} v_{1}\right)}{u_{1}(2 c d+(c+d) u v)} v_{x} .
\end{aligned}\right.
$$

This system possesses two independent non-trivial n-integrals (1.9) and (1.10). One can confirm that this system possesses also the following two n-integrals

$$
I_{2}^{*}=\frac{(2 c d+(c+d) u v) u_{x}}{u(u v+d)}, \quad J_{2}^{*}=\frac{(c-d) u v^{2} u_{x}}{2 c(u v+d)(2 c d+(c+d) u v)}+\frac{u v_{x}}{2 c d+(c+d) u v} .
$$

Considering the corresponding $x$-ring we can also find the $x$-integrals given by

$$
\mathcal{F}_{1}=\frac{u_{1}}{u}\left(\frac{2 c d+(c+d) u v}{2 c d+(c+d) u_{1} v_{1}}\right)^{\frac{c-d}{c+d}}, \quad \mathcal{F}_{2}=\frac{u_{1} v_{1}-u v}{u_{2} v_{2}-u v} .
$$

Example 1.6. Let $K_{2}=0$, then

$$
\mathcal{D}_{2}=\frac{(c+d) u_{1} v_{1}}{2 c d+(c+d) u_{1} v_{1}} .
$$

Using (1.23) we get the system

$$
\left\{\begin{aligned}
u_{1 x}= & \frac{(c+d) u_{1} v\left(u_{1} v_{1}+d\right)}{(u v+d)\left(2 c d+(c+d) u_{1} v_{1}\right)} u_{x} \\
v_{1 x}= & \frac{(d-c) v}{2 c(u v+d)}\left(\frac{(c+d) u_{1} v_{1}^{2}}{2 c d+(c+d) u_{1} v_{1}}-\frac{2 c d+(c+d) u_{1} v_{1}}{(c+d) u_{1}}\right) u_{x} \\
& +\frac{2 c d+(c+d) u_{1} v_{1}}{(c+d) u_{1} v} v_{x} .
\end{aligned}\right.
$$

This system possesses two independent non-trivial n-integrals (1.9) and (1.10) and two independent $x$-integrals

$$
\mathcal{F}_{1}=\frac{1}{c+d}\left(\frac{2 c d+(c+d) u_{1} v_{1}}{v u_{1}}\right)^{\frac{c+d}{2 d}}+\frac{u}{u_{1}}\left(\frac{2 c d+(c+d) u_{1} v_{1}}{v u_{1}}\right)^{\frac{c-d}{2 d}}
$$

and

$$
\mathcal{F}_{2}=\frac{v_{1} u_{1}^{\frac{d-c}{2 d}}\left(2 c d+(c+d) u_{1} v_{1}\right)^{\frac{c+d}{2 d}}}{v^{\frac{c+d}{2 d}}\left(2 c d+(c+d)\left(u_{1} v_{1}+u_{2} v_{2}\right)\right)} .
$$

Remark 1.3. We consider a function $\mathcal{D}_{2}$ defined implicitly by

$$
H\left(K_{2}, L_{2}\right)=L_{2}-(2 c d)^{2 d /(c+d)}=0
$$

and expand it into a series of the form

$$
\mathcal{D}_{2}\left(u_{1}, v, v_{1}\right)=\sum_{n=0}^{\infty} a_{n}\left(v_{1}-v\right)^{n}
$$


where coefficients $a_{n}$ depend on variables $u_{1}$ and $v$ only. This gives:

$$
\mathcal{D}_{2}\left(u_{1}, v, v_{1}\right)=1+\frac{c}{v(u v+c)}\left(v_{1}-v\right)+\sum_{n=2}^{\infty} a_{n}\left(v_{1}-v\right)^{n} .
$$

By letting $u_{1}=u+\varepsilon u_{y}, v_{1}=v+\varepsilon v_{y}$ and passing to the limit as $\varepsilon \rightarrow 0$ one can see that system (1.23) becomes (1.8) with $\alpha=-1$.

Theorem 1.3. System (1.13) admits n-integrals (1.11) and (1.12) if and only if it is of the form

$$
\left\{\begin{array}{l}
u_{1 x}=\frac{u_{1} v_{1}+d_{1}}{\mathcal{D}_{3}(u v+d)} u_{x}, \\
v_{1 x}=\left(\frac{-\beta v_{1}^{2}}{\mathcal{D}_{3}(u v+d)}+\frac{\beta v^{2} \mathcal{D}_{3}^{\beta}}{u v+d}\right) u_{x}+\mathcal{D}_{3}^{\beta} v_{x} .
\end{array}\right.
$$

The function $\mathcal{D}_{3}$ is given implicitly by $H\left(n, K_{3}, L_{3}\right)=0$, where, for each $n$, the symbol $H$ denotes an arbitrary smooth function and

$$
\begin{gathered}
K_{3}=\frac{\left(v_{1}-v \mathcal{D}_{3}^{\beta}\right)^{\beta^{-1}}\left(d_{1} u-d u_{1} \mathcal{D}_{3}\right)}{\mathcal{D}_{3}} \\
L_{3}=\left(v_{1}-v \mathcal{D}_{3}^{\beta}\right)^{(1-\beta) \beta^{-1}}\left(d_{1} \mathcal{D}_{3}^{\beta-1}-d_{1}+(\beta-1) u_{1}\left(v_{1}-v \mathcal{D}_{3}^{\beta}\right)\right) .
\end{gathered}
$$

Here $d_{1}=D d$ and $D$ is the shift operator.

Example 1.7. Considering $K_{3}=0$, we find

$$
\mathcal{D}_{3}=\frac{v_{1}^{1 / \beta}}{v^{1 / \beta}} .
$$

Using (1.26), we get the system

$$
\left\{\begin{array}{l}
u_{1 x}=\frac{\left(u_{1} v_{1}+d_{1}\right) v^{1 / \beta}}{(u v+d) v_{1}^{1 / \beta}} u_{x} \\
v_{1 x}=\left(-\frac{\beta v_{1}^{2} v^{1 / \beta}}{v_{1}^{1 / \beta}(u v+d)}+\frac{\beta v^{2} v_{1}}{v(u v+d)}\right) u_{x}+\frac{v_{1}}{v} v_{x} .
\end{array}\right.
$$

This system possesses two independent non-trivial n-integrals (1.11) and (1.12) and two independent $x$-integrals

$$
\mathcal{F}_{1}=\left(1-\left(\frac{v_{1}}{v}\right)^{\frac{1-\beta}{\beta}}\right)\left(-d_{1} u+d u_{1}\left(\frac{v_{1}}{v}\right)^{\frac{1}{\beta}}\right)^{\beta-1}
$$

and

$$
\mathcal{F}_{2}=\frac{v^{\frac{1-\beta}{\beta}}-v_{2}^{\frac{1-\beta}{\beta}}}{v^{\frac{1-\beta}{\beta}}-v_{1}^{\frac{1-\beta}{\beta}}} .
$$

One can confirm that this system possesses also the following two n-integrals

$$
I_{3}^{*}=\frac{v^{1 / \beta} u_{x}}{u v+d}, \quad J_{3}^{*}=\frac{v_{x}}{v}+\frac{\beta v u_{x}}{u v+d} .
$$

Example 1.8. Let $K_{3}=0$, we find

$$
\mathcal{D}_{3}=\frac{d_{1} u}{d u_{1}} \text {. }
$$


By 1.26 we get the system

$$
\left\{\begin{array}{l}
u_{1 x}=\frac{\left(u_{1} v_{1}+d_{1}\right) d u_{1}}{(u v+d) d_{1} u} u_{x} \\
v_{1 x}=\left(-\frac{\beta d v_{1}^{2} u_{1}}{d_{1} u(u v+d)}+\frac{\beta d_{1}^{\beta} v^{2} u^{\beta}}{d^{\beta} u_{1}^{\beta}(u v+d)}\right) u_{x}+\frac{d_{1}^{\beta} u^{\beta}}{d^{\beta} u_{1}^{\beta}} v_{x}
\end{array}\right.
$$

This system possesses two independent non-trivial n-integrals (1.11) and (1.12) and two independent $x$-integrals

and

$$
\mathcal{F}_{1}=\frac{d_{1}^{\beta} u^{\beta} v-d^{\beta} u_{1}^{\beta} v_{1}}{d_{2}^{\beta} u_{1}^{\beta} v_{1}-d_{1}^{\beta} u_{2}^{\beta} v_{2}}
$$

$$
\mathcal{F}_{2}=\frac{\left(d_{1}^{\beta} u^{\beta} v-d^{\beta} u_{1}^{\beta} v_{1}\right)\left(d d_{1}^{\beta} u^{\beta} u-d_{1} d^{\beta} u_{1}^{\beta} u+(1-\beta) u u_{1}\right)}{d d_{1} u u_{1}} .
$$

We confirm that this system possesses also the following two n-integrals

$$
I_{3}^{* *}=\frac{d u_{x}}{u(u v+d)}, \quad J_{3}^{* *}=\frac{u^{\beta} v_{x}}{d^{\beta}}+\frac{\beta v^{2} u^{\beta} u_{x}}{d^{\beta}(u v+d)} .
$$

Example 1.9. Considering $L_{3}=0$ with $\beta=2$, we get $\mathcal{D}_{3}=\frac{d_{1}+R}{2 u_{1} v}$, where

$$
R=\sqrt{d_{1}^{2}+4 u_{1} v\left(u_{1} v_{1}-d_{1}\right)} .
$$

Then by 1.26 we arrive at the system

$$
\left\{\begin{aligned}
u_{1 x}= & \frac{\left(u_{1} v_{1}+d_{1}\right)\left(d_{1}-R\right)}{2(u v+d)\left(d_{1}-u_{1} v_{1}\right)} u_{x} \\
v_{1 x}= & \left(\frac{v_{1}^{2}\left(R-d_{1}\right)}{d_{1}-u_{1} v_{1}}+\frac{d_{1}^{2}+2 u_{1} v\left(u_{1} v_{1}-d_{1}\right)+d_{1} R}{u_{1}^{2}}\right) \frac{u_{x}}{u v+d} \\
& +\frac{d_{1}^{2}+2 u_{1} v\left(u_{1} v_{1}-d_{1}\right)+d_{1} R}{2 u_{1}^{2} v^{2}} v_{x} .
\end{aligned}\right.
$$

This system possesses two independent non-trivial n-integrals 1.11) and 1.12.

Example 1.10. Considering $L_{3}=0$ with $\beta=1 / 2$, we see that

$$
\mathcal{D}_{3}^{1 / 2}=\frac{2 d_{1}+u_{1} v_{1}+R}{2 u_{1} v}
$$

where

$$
R=\sqrt{\left(2 d_{1}+u_{1} v_{1}\right)^{2}-8 d_{1} u_{1} v} .
$$

Employing (1.26), we get the system

$$
\left\{\begin{array}{l}
u_{1 x}=\frac{\left(u_{1} v_{1}+d_{1}\right)\left(2 d_{1}+u_{1} v_{1}-R\right)^{2}}{16 d_{1}^{2}(u v+d)} u_{x} \\
v_{1 x}=\left(\frac{-v_{1}^{2}\left(2 d_{1}+u_{1} v_{1}-R\right)^{2}}{32 d_{1}^{2}}+\frac{v\left(2 d_{1}+u_{1} v_{1}+R\right)}{4 u_{1}}\right) \frac{u_{x}}{u v+d}+\frac{2 d_{1}+u_{1} v_{1}+R}{2 u_{1} v} v_{x} .
\end{array}\right.
$$

This system possesses two independent non-trivial n-integrals (1.11) and (1.12).

Remark 1.4. In both previous examples the corresponding $x$-rings have the following multiplication table 


\begin{tabular}{c|c|c|c}
{$\left[E_{i}, E_{j}\right]$} & $E_{1}$ & $E_{2}$ & $E_{3}$ \\
\hline$E_{1}$ & 0 & $E_{3}$ & $\frac{-2 v}{d+u v} E_{3}$ \\
\hline$E_{2}$ & $-E_{3}$ & 0 & $\frac{-2 u}{d+u v} E_{3}$ \\
\hline$E_{3}$ & $\frac{2 v}{d+u v} E_{3}$ & $\frac{2 u}{d+u v} E_{3}$ & 0
\end{tabular}

where the fields $X, Y_{1}, Y_{2}, E_{1}, E_{2}$ and $E_{3}$ are introduced in the same way as in Remark 1. This shows that the $x$-rings are finite-dimensional and the corresponding systems (1.31) and (1.32) are Darboux integrable.

Remark 1.5. We consider a function $\mathcal{D}_{3}$ given implicitly by $H\left(K_{3}, L_{3}\right)=L_{3}=0$ and expand it into a series of the form

$$
\mathcal{D}_{3}\left(u_{1}, v, v_{1}\right)=\sum_{n=0}^{\infty} a_{n}\left(v_{1}-v\right)^{n}
$$

where coefficients $a_{n}$ depend on variables $u_{1}$ and $v$ only. Then

$$
\mathcal{D}_{3}\left(u_{1}, v, v_{1}\right)=1+\frac{u_{1}}{\beta u_{1} v-d_{1}}\left(v_{1}-v\right)+\sum_{n=2}^{\infty} a_{n}\left(v_{1}-v\right)^{n} .
$$

By letting $u_{1}=u+\varepsilon u_{y}, v_{1}=v+\varepsilon v_{y}$ and passing to the limit as $\varepsilon \rightarrow 0$ one can see that system (1.26) becomes (1.8). We observe that $\beta=-\alpha$ and constants $\alpha, c, d$ satisfy the identity $d=\alpha c$.

\section{Proof of Theorem 1.1}

It follows from the identity $D J_{1}=J_{1}$ that

$$
\frac{u_{1 x x}}{u_{1 x}}-\left(1+\frac{1}{\alpha_{1}}\right) \frac{u_{1 x}}{u_{1}+v_{1}}-\frac{v_{1 x}}{u_{1}+v_{1}}=\frac{u_{x x}}{u_{x}}-\frac{(\alpha+1) u_{x}+\alpha v_{x}}{\alpha(u+v)},
$$

that is

$$
\begin{aligned}
\frac{f_{x}+f_{u} u_{x}+f_{v} v_{x}+f_{u_{1}} f+f_{v_{1}} g+f_{u_{x}} u_{x x}+f_{v_{x}} v_{x x}}{f} & -\left(1+\frac{1}{\alpha_{1}}\right) \frac{f}{u_{1}+v_{1}} \\
& -\frac{g}{u_{1}+v_{1}}=\frac{u_{x x}}{u_{x}}-\frac{(\alpha+1) u_{x}+\alpha v_{x}}{\alpha(u+v)} .
\end{aligned}
$$

By comparing the coefficients at $v_{x x}$ and $u_{x x}$, we get

$$
f_{v_{x}}=0 \quad \text { and } \quad \frac{f_{u_{x}}}{f}=\frac{1}{u_{x}} .
$$

Hence,

$$
f\left(x, n, u, v, u_{1}, v_{1}, u_{x}, v_{x}\right)=A\left(x, n, u, v, u_{1}, v_{1}\right) u_{x} .
$$

It follows from $D I_{1}=I_{1}$ that

$$
\left(1+\frac{1}{\alpha_{1}}\right) v_{1}\left(\frac{A u_{x}}{u_{1}+v_{1}}\right)^{1-\alpha_{1}}-g\left(\frac{A u_{x}}{u_{1}+v_{1}}\right)^{-\alpha_{1}}=\left(1+\frac{1}{\alpha}\right) v\left(\frac{u_{x}}{u+v}\right)^{1-\alpha}-v_{x}\left(\frac{u_{x}}{u+v}\right)^{-\alpha} \text {. }
$$

We first consider the case $\alpha_{1} \neq \alpha$. We have:

$$
g=T u_{x}+M u_{x}^{1+\alpha_{1}-\alpha}+N v_{x} u_{x}^{\alpha_{1}-\alpha},
$$

where

$$
T=\left(1+\frac{1}{\alpha_{1}}\right) \frac{v_{1} A}{u_{1}+v_{1}}, \quad M=-\left(1+\frac{1}{\alpha}\right) \frac{v(u+v)^{\alpha-1} A^{\alpha_{1}}}{\left(u_{1}+v_{1}\right)^{\alpha_{1}}}, \quad N=\frac{(u+v)^{\alpha} A^{\alpha_{1}}}{\left(u_{1}+v_{1}\right)^{\alpha_{1}}} .
$$


Substituting the expression for $g$ and $f$ into 2.1) and comparing the coefficients at $u_{x}^{0}, u_{x}, v_{x}$, $u_{x}^{1+\alpha_{1}-\alpha}$ and $v_{x} u_{x}^{\alpha_{1}-\alpha}$, we get

$$
\begin{aligned}
& \frac{A_{x}}{A}=0 \\
& \frac{A_{u}}{A}+A_{u_{1}}+\frac{A_{v_{1}}}{A} T-\left(1+\frac{1}{\alpha_{1}}\right) \frac{A}{u_{1}+v_{1}}-\frac{T}{u_{1}+v_{1}}=-\left(1+\frac{1}{\alpha}\right) \frac{1}{u+v}, \\
& \frac{A_{v}}{A}=-\frac{1}{u+v}, \\
& M\left(\frac{A_{v_{1}}}{A}-\frac{1}{u_{1}+v_{1}}\right)=0 \\
& N\left(\frac{A_{v_{1}}}{A}-\frac{1}{u_{1}+v_{1}}\right)=0 .
\end{aligned}
$$

It follows from (2.5)- 2.7$)$ that

$$
A=\frac{u_{1}+v_{1}}{u+v} S\left(n, u, u_{1}\right)
$$

where $S\left(n, u, u_{1}\right)$ is a function depending on $n, u, u_{1}$ only. Substitute this expression for $A$ into (2.4), we find that

$$
(u+v)\left(u_{1}+v_{1}\right) \frac{S_{u}}{S}+\left(u_{1}+v_{1}\right)^{2} S_{u_{1}}+\left(v_{1}-\frac{u_{1}}{\alpha_{1}}-\frac{\left(1+\alpha_{1}\right) v_{1}\left(u_{1}+v_{1}\right)^{2}}{\alpha_{1}}\right) S+\frac{u_{1}+v_{1}}{\alpha}=0 .
$$

Differentiating the last equation three times with respect to $v_{1}$, we get

$$
-6\left(1+\frac{1}{\alpha_{1}}\right) S=0, \quad \text { hence, } \quad S=0 .
$$

Hence, $A=0$ is the only solution when $\alpha \neq \alpha_{1}$.

Now we consider the case when $\alpha$ is a constant, that is $\alpha$ is independent of $n$. We have:

$$
g=\left(1+\frac{1}{\alpha}\right)\left(\frac{A v_{1}}{u_{1}+v_{1}}-\frac{v A^{\alpha}}{u+v}\left(\frac{u+v}{u_{1}+v_{1}}\right)^{\alpha}\right) u_{x}+\left(A \frac{u+v}{u_{1}+v_{1}}\right)^{\alpha} v_{x} .
$$

We substitute the expressions for $f$ and $g$ into (2.1) and compare the coefficients at $v_{x}, u_{x}$ and the free term. This gives:

$$
\begin{aligned}
& \frac{A_{x}}{A}=0, \\
& \frac{A_{u}}{A}+A_{u_{1}}+\left(1+\frac{1}{\alpha}\right)\left[A_{v_{1}} \frac{v_{1}}{u_{1}+v_{1}}-\frac{v A^{\alpha} A_{v_{1}}}{A(u+v)}\left(\frac{u+v}{u_{1}+v_{1}}\right)^{\alpha}-\frac{A}{u_{1}+v_{1}}\right. \\
& \left.-\frac{A v_{1}}{\left(u_{1}+v_{1}\right)^{2}}+\frac{v A^{\alpha}}{(u+v)\left(u_{1}+v_{1}\right)}\left(\frac{u+v}{u_{1}+v_{1}}\right)^{\alpha}+\frac{1}{u+v}\right]=0, \\
& \frac{A_{v}}{A}+\frac{A_{v_{1}} A^{\alpha}}{A}\left(\frac{u+v}{u_{1}+v_{1}}\right)^{\alpha}-\frac{A^{\alpha}}{u_{1}+v_{1}}\left(\frac{u+v}{u_{1}+v_{1}}\right)^{\alpha}+\frac{1}{u+v}=0 .
\end{aligned}
$$

Let

$$
\mathcal{D}_{1}=\left(\frac{u+v}{u_{1}+v_{1}}\right)^{\alpha} A^{\alpha} .
$$

In terms of the function $\mathcal{D}_{1}$, the equations 2.11$)$ and $(2.12)$ cast into the form

$$
\begin{gathered}
(u+v) \mathcal{D}_{1 u}+\left(u_{1}+v_{1}\right) \mathcal{D}_{1}^{\alpha^{-1}} \mathcal{D}_{1 u_{1}}+\frac{\alpha+1}{\alpha}\left(v_{1} \mathcal{D}_{1}^{\alpha^{-1}}-v \mathcal{D}_{1}\right) \mathcal{D}_{1 v_{1}}-\mathcal{D}_{1}\left(\mathcal{D}_{1}^{\alpha^{-1}}-1\right)=0, \\
\frac{\mathcal{D}_{1 v}}{\mathcal{D}_{1}}+\mathcal{D}_{1 v_{1}}=0
\end{gathered}
$$


The set of solutions of the above system is not empty. For example, $\mathcal{D}_{1}=1$ is a singular solution leading to Darboux integrable system (1.17). Let $\mathcal{D}_{1} \neq 1$. It is convenient to regard $\mathcal{D}_{1}$ as a function of $n, u, v, u_{1}, v_{1}$ defined implicitly by the equation as follows

$$
W\left(n, u, v, u_{1}, v_{1}, \mathcal{D}_{1}\right)=0 .
$$

Then in terms of function $W=W\left(n, u, v, u_{1}, v_{1}, \mathcal{D}_{1}\right)$, equations (2.14) and (2.15) can be rewritten as

$$
\begin{aligned}
& (u+v) W_{u}+\left(u_{1}+v_{1}\right) \mathcal{D}_{1}^{\alpha^{-1}} W_{u_{1}}+\frac{\alpha+1}{\alpha}\left(v_{1} \mathcal{D}_{1}^{\alpha^{-1}}-v \mathcal{D}_{1}\right) W_{v_{1}}+\mathcal{D}_{1}\left(\mathcal{D}_{1}^{\alpha^{-1}}-1\right) W_{\mathcal{D}_{1}}=0, \\
& \frac{W_{v}}{\mathcal{D}_{1}}+W_{v_{1}}=0 .
\end{aligned}
$$

Under the change of variables

$$
\tilde{v}=v, \quad \tilde{v}_{1}=v_{1}-v \mathcal{D}_{1}, \quad \tilde{u}=u, \quad \tilde{u}_{1}=u_{1}, \quad \tilde{\mathcal{D}}_{1}=\mathcal{D}_{1},
$$

the above equations cast into the form

$$
\begin{aligned}
(\tilde{u}+\tilde{v}) W_{\tilde{u}}+\left(\tilde{u}_{1}+\tilde{v}_{1}+\tilde{v} \tilde{\mathcal{D}}_{1}\right) \tilde{\mathcal{D}}_{1}^{\alpha^{-1}} W_{\tilde{u}_{1}} & +\left(\left(1+\frac{1}{\alpha}\right) \tilde{v}_{1} \tilde{\mathcal{D}}_{1}^{\alpha^{-1}}+\frac{1}{\alpha} \tilde{v}\left(\tilde{\mathcal{D}}_{1}^{1+\alpha^{-1}}-\tilde{\mathcal{D}}_{1}\right)\right) W_{\tilde{v}_{1}} \\
& +\left(\tilde{\mathcal{D}}_{1}^{1+\alpha^{-1}}-\tilde{\mathcal{D}}_{1}\right) W_{\tilde{\mathcal{D}}_{1}}=0, \\
W_{\tilde{v}}=0 . &
\end{aligned}
$$

We differentiate the first equation with respect to $\tilde{v}$, then use the identity $W_{\tilde{v}}=0$, and get two new equations

$$
\begin{aligned}
& W_{\tilde{u}}+\tilde{\mathcal{D}}_{1}^{1+\alpha^{-1}} W_{\tilde{u}_{1}}+\frac{1}{\alpha}\left(\tilde{\mathcal{D}}_{1}^{1+\alpha^{-1}}-\tilde{\mathcal{D}}_{1}\right) W_{\tilde{v}_{1}}=0, \\
& \tilde{u} W_{\tilde{u}}+\left(\tilde{u}_{1}+\tilde{v}_{1}\right) \tilde{\mathcal{D}}_{1}^{\alpha^{-1}} W_{\tilde{u}_{1}}+\frac{\alpha+1}{\alpha} \tilde{v}_{1} \tilde{\mathcal{D}}_{1}^{\alpha^{-1}} W_{\tilde{v}_{1}}+\left(\tilde{\mathcal{D}}_{1}^{1+\alpha^{-1}}-\tilde{\mathcal{D}}_{1}\right) W_{\tilde{\mathcal{D}}_{1}}=0 .
\end{aligned}
$$

In the latter system, we make the change of variables

$$
u_{1}^{*}=\tilde{u}_{1}-\tilde{\mathcal{D}}_{1}^{1+\alpha^{-1}} \tilde{u}, \quad v_{1}^{*}=\alpha \tilde{\mathcal{D}}_{1}^{\alpha^{-1}} \tilde{v}_{1}+\left(1-\tilde{\mathcal{D}}_{1}^{\alpha^{-1}}\right) \tilde{u}_{1}, \quad u^{*}=\tilde{u}, \quad v^{*}=\tilde{v}, \quad \mathcal{D}_{1}^{*}=\tilde{\mathcal{D}}_{1}
$$

and we get:

$$
\begin{gathered}
W_{u^{*}}=0 \\
\left(\left(\mathcal{D}_{1}^{* \alpha^{-1}}+\alpha^{-1}\left(1-\mathcal{D}_{1}^{*-\alpha^{-1}}\right)\right) u_{1}^{*}+\alpha^{-1} v_{1}^{*} \mathcal{D}_{1}^{*-\alpha^{-1}}\right) W_{u_{1}^{*}} \\
+\frac{\alpha+1}{\alpha} v_{1}^{*} \mathcal{D}_{1}^{* \alpha^{-1}} W_{v^{*}}+\mathcal{D}_{1}^{*}\left(\mathcal{D}_{1}^{* \alpha^{-1}}-1\right) W_{\mathcal{D}_{1}^{*}}=0 .
\end{gathered}
$$

The last equation has a general solution $H\left(n, K_{1}, L_{1}\right)=0$, where $K_{1}, L_{1}$ rewritten in old variables are given by (1.15), (1.16) and $H$ is a smooth function for each $n$. Now, using identities (2.13), (2.2) and (2.9), we obtain system (1.14). This completes the proof.

\section{Proof of Theorem 1.2}

The identity $D J_{2}=J_{2}$ implies that

$$
\begin{aligned}
\frac{f_{x}+f_{u} u_{x}+f_{v} v_{x}+f_{u_{1}} f+f_{v_{1}} g+f_{u_{x}} u_{x x}+f_{v_{x}} v_{x x}}{f} & +\frac{\left(d_{1}-c_{1}\right) v_{1} f-c_{1} u_{1} g}{c_{1}\left(u_{1} v_{1}+d_{1}\right)} \\
& =\frac{u_{x x}}{u_{x}}+\frac{(d-c) v u_{x}-c u v_{x}}{c(u v+d)} .
\end{aligned}
$$

Comparing the coefficients at $u_{x x}$ and $v_{x x}$ in the above identity, we get

$$
f_{v_{x}}=0 \quad \text { and } \quad \frac{f_{u_{x}}}{f}=\frac{1}{u_{x}} .
$$


Hence,

The identity $D I_{2}=I_{2}$ implies

$$
f=A\left(x, n, u, v, u_{1}, v_{1}\right) u_{x} .
$$

$$
\frac{\left(d_{1}-c_{1}\right) v_{1}^{2} A^{2} u_{x}}{2\left(u_{1} v_{1}+d_{1}\right)^{2}}-\frac{c A g}{u_{1} v_{1}+d_{1}}=\frac{(d-c) v^{2} u_{x}}{2(u v+d)^{2}}-\frac{c v_{x}}{u v+d} .
$$

It follows from $(3.3)$ that

$$
g=\left(\frac{\left(d_{1}-c_{1}\right) v_{1}^{2} A}{2 c_{1}\left(u_{1} v_{1}+d_{1}\right)}-\frac{(d-c) v_{1}^{2}\left(u_{1} v_{1}+d_{1}\right)}{2 c_{1} A(u v+d)^{2}}\right) u_{x}+\frac{c\left(u_{1} v_{1}+d_{1}\right)}{c_{1} A(u v+d)} v_{x} .
$$

Substituting the expressions for $f$ and $g$ into (3.1) and comparing the coefficients at $u_{x}, v_{x}$ and the free term, we get

$$
\begin{aligned}
& \frac{A_{x}}{A}=0, \\
& \begin{aligned}
\frac{A_{u}}{A}+A_{u_{1}} & +\left(\frac{A_{v_{1}}}{A}-\frac{u_{1}}{u_{1} v_{1}+d_{1}}\right)\left(\frac{\left(d_{1}-c_{1}\right) v_{1}^{2} A}{2 c_{1}\left(u_{1} v_{1}+d_{1}\right)}-\frac{(d-c) v^{2}\left(u_{1} v_{1}+d_{1}\right)}{2 c_{1} A(u v+d)^{2}}\right) \\
& \quad+\frac{\left(d_{1}-c_{1}\right) v_{1} A}{c_{1}\left(u_{1} v_{1}+d_{1}\right)}-\frac{(d-c) v}{c(u v+d)}=0, \\
& \frac{A_{v}}{A}+\frac{c\left(u_{1} v_{1}+d_{1}\right)}{c_{1} A(u v+d)}\left(\frac{A_{v_{1}}}{A}-\frac{u_{1}}{u_{1} v_{1}+d_{1}}\right)+\frac{u}{u v+d}=0 .
\end{aligned}
\end{aligned}
$$

One can check that

$$
A=\frac{v\left(u_{1} v_{1}+d\right)}{v_{1}(u v+d)}
$$

is a particular solution provided $d_{1}=d$ and $c_{1}=c$.

Now assuming that

we introduce a new function

$$
A \neq \frac{v\left(u_{1} v_{1}+d\right)}{v_{1}(u v+d)}
$$

$$
\mathcal{D}_{2}=\frac{v_{1}(u v+d)}{v\left(u_{1} v_{1}+d_{1}\right)} A .
$$

In terms of $\mathcal{D}_{2}$, system $(3.6)$ becomes

$$
\begin{aligned}
& \mathcal{D}_{2 x}=0 \\
& (u v+d) \mathcal{D}_{2 u}+\frac{v\left(u_{1} v_{1}+d_{1}\right) \mathcal{D}_{2}}{v_{1}} \mathcal{D}_{2 u_{1}}+\frac{v v_{1}}{2 c_{1}}\left(\left(d_{1}-c_{1}\right) \mathcal{D}_{2}-(d-c) \mathcal{D}_{2}{ }^{-1}\right) \mathcal{D}_{2 v_{1}} \\
& -\frac{d v}{c} \mathcal{D}_{2}+\frac{\left(d_{1}+c_{1}\right) v}{2 c_{1}} \mathcal{D}_{2}^{2}+\frac{v(d-c)}{2 c_{1}}=0, \\
& c_{1} v \mathcal{D}_{2} \mathcal{D}_{2 v}+c v_{1} \mathcal{D}_{2 v_{1}}+\left(-c \mathcal{D}_{2}+c_{1} \mathcal{D}_{2}^{2}\right)=0 .
\end{aligned}
$$

In the same way as in the proof of Theorem 1.1, we introduce a function $W\left(n, u, v, u_{1}, v_{1}, \mathcal{D}_{2}\right)$. For the function $W=W\left(n, u, v, u_{1}, v_{1}, \mathcal{D}_{2}\right)$ the last two equations become

$$
\begin{gathered}
(u v+d) W_{u}+\frac{v\left(u_{1} v_{1}+d_{1}\right)}{v_{1}} \mathcal{D}_{2} W_{u_{1}}+\frac{v v_{1}}{2 c_{1}}\left(\left(d_{1}-c_{1}\right) \mathcal{D}_{2}-(d-c) \mathcal{D}_{2}{ }^{-1}\right) W_{v_{1}} \\
+\left(\frac{d v}{c} \mathcal{D}_{2}-\frac{\left(d_{1}+c_{1}\right) v}{2 c_{1}} \mathcal{D}_{2}{ }^{2}-\frac{v(d-c)}{2 c_{1}}\right) W_{\mathcal{D}_{2}}=0, \\
c_{1} v \mathcal{D}_{2} W_{v}+c v_{1} W_{v_{1}}+\left(c \mathcal{D}_{2}-c_{1} \mathcal{D}_{2}{ }^{2}\right) W_{\mathcal{D}_{2}}=0 .
\end{gathered}
$$

In new variables

$$
\tilde{u}=u, \quad \tilde{u}_{1}=u_{1}, \quad \tilde{v}=v\left(c_{1} \mathcal{D}_{2}-c\right), \quad \tilde{v}_{1}=v_{1}\left(c_{1} \mathcal{D}_{2}-c\right) \mathcal{D}_{2}^{-1}, \quad \tilde{\mathcal{D}}_{2}=\mathcal{D}_{2},
$$


the last system can be rewritten as

$$
\begin{aligned}
\left(\left(c_{1} \tilde{\mathcal{D}}_{2}-c\right) \tilde{u} \tilde{v}\right. & \left.+d\left(c_{1} \tilde{\mathcal{D}}_{2}-c\right)^{2}\right) W_{\tilde{u}}+\frac{\tilde{v}}{\tilde{v}_{1}}\left(\tilde{u}_{1} \tilde{v}_{1} \tilde{\mathcal{D}}_{2}\left(c_{1} \tilde{\mathcal{D}}_{2}-c\right)+d_{1}\left(c_{1} \tilde{\mathcal{D}}_{2}-c\right)^{2}\right) W_{\tilde{u}_{1}} \\
& +\tilde{v}^{2}\left(\frac{c_{1} d \tilde{\mathcal{D}}_{2}}{c}-\frac{\left(d_{1}+c_{1}\right) \tilde{\mathcal{D}}_{2}^{2}}{2}+\frac{c-d}{2}\right) W_{\tilde{v}} \\
& +\tilde{v} \tilde{v}_{1}\left(\frac{\left(d_{1}-c_{1}\right) \tilde{\mathcal{D}}_{2}^{2}}{2}-\frac{c d_{1} \tilde{\mathcal{D}}_{2}}{c_{1}}+\frac{c+d}{2}\right) W_{\tilde{v}_{1}}=0,
\end{aligned}
$$

$W_{\tilde{\mathcal{D}}_{2}}=0$

Special solutions of 3.9 may exist only when $\tilde{\mathcal{D}}_{2}=c_{1} / c$. We differentiate equation 3.9 with respect to $\tilde{\mathcal{D}}_{2}$ three times and get the following system of three equations

$$
\begin{aligned}
& \left(d c^{2}-c \tilde{u} \tilde{v}\right) W_{\tilde{u}}+c^{2} d_{1} \frac{\tilde{v}}{\tilde{v}_{1}} W_{\tilde{u}_{1}}+\frac{(c-d) \tilde{v}^{2}}{2} W_{\tilde{v}}+\frac{(c+d) \tilde{v} \tilde{v}_{1}}{2} W_{\tilde{v}_{1}}=0, \\
& \left(c_{1} \tilde{u} \tilde{v}-2 d c_{1} c\right) W_{\tilde{u}}-\left(2 d_{1} c_{1} c \frac{\tilde{v}}{\tilde{v}_{1}}+c \tilde{u}_{1} \tilde{v}\right) W_{\tilde{u}_{1}}+\frac{c_{1} d \tilde{v}^{2}}{c} W_{\tilde{v}}-\frac{c d_{1} \tilde{v} \tilde{v}_{1}}{c_{1}} W_{\tilde{v}_{1}}=0, \\
& d c_{1}^{2} W_{\tilde{u}}+\left(\frac{d_{1} c_{1}^{2} \tilde{v}}{\tilde{v}_{1}}+c_{1} \tilde{u}_{1} \tilde{v}\right) W_{\tilde{u}_{1}}-\frac{\left(d_{1}+c_{1}\right) \tilde{v}^{2}}{2} W_{\tilde{v}}+\frac{\left(d_{1}-c_{1}\right) \tilde{v} \tilde{v}_{1}}{2} W_{\tilde{v}_{1}}=0,
\end{aligned}
$$

that has no solutions if $c_{1} \neq c$ or $d_{1} \neq d$. In the case $c_{1}=c$ and $d_{1}=d$ the system becomes

$$
\begin{aligned}
& W_{\tilde{u}}-\frac{\tilde{v}^{2}\left(2 c^{2} d+(c-d) \tilde{u}_{1} \tilde{v}_{1}\right)}{2 c\left(\tilde{u} \tilde{u}_{1} \tilde{v} \tilde{v}_{1}+c d\left(\tilde{u} \tilde{v}-\tilde{u}_{1} \tilde{v}_{1}\right)\right)} W_{\tilde{v}}-\frac{\tilde{v} \tilde{v}_{1}\left(2 c^{2} d+(c+d) \tilde{u}_{1} \tilde{v}_{1}\right)}{2 c\left(\tilde{u} \tilde{u}_{1} \tilde{v} \tilde{v}_{1}+c d\left(\tilde{u} \tilde{v}-\tilde{u}_{1} \tilde{v}_{1}\right)\right)} W_{\tilde{v}_{1}}=0, \\
& W_{\tilde{u}_{1}}-\frac{\tilde{v} \tilde{v}_{1}\left(-2 c^{2} d+(c+d) \tilde{u} \tilde{v}\right)}{2 c\left(\tilde{u} \tilde{u}_{1} \tilde{v} \tilde{v}_{1}+c d\left(\tilde{u} \tilde{v}-\tilde{u}_{1} \tilde{v}_{1}\right)\right)} W_{\tilde{v}}+\frac{\tilde{v}_{1}^{2}\left(2 c^{2} d+(-c+d) \tilde{u} \tilde{v}\right)}{2 c\left(\tilde{u} \tilde{u}_{1} \tilde{v} \tilde{v}_{1}+c d\left(\tilde{u} \tilde{v}-\tilde{u}_{1} \tilde{v}_{1}\right)\right)} W_{\tilde{v}_{1}}=0 .
\end{aligned}
$$

Under the change of variables

$$
\begin{aligned}
& u_{1}^{*}=\tilde{u}_{1}, \quad v_{1}^{*}=\tilde{v}_{1}, \quad v^{*}=\frac{\tilde{v}}{\tilde{v}_{1}}\left(2 c^{2} d+(c+d) \tilde{u}_{1} \tilde{v}_{1}\right)^{\frac{2 d}{c+d}}, \\
& u^{*}=\tilde{u} \tilde{v}_{1}\left(2 c^{2} d+(c+d) \tilde{u}_{1} \tilde{v}_{1}\right)^{\frac{c-d}{c+d}}-2 c^{2} d \tilde{u}_{1} \tilde{v}_{1}^{2} \tilde{v}^{-1}\left(2 c^{2} d+(c+d) \tilde{u}_{1} \tilde{v}_{1}\right)^{-\frac{2 d}{c+d}},
\end{aligned}
$$

equations (3.13) and (3.14) become $W_{v_{1}^{*}}=0$ and $W_{u_{1}^{*}}=0$, respectively. We rewrite these first integrals in old variables and get the general solution in an implicit form $H\left(n, K_{2}, L_{2}\right)=0$, where, for each $n, H$ is a smooth function and $K_{2}, L_{2}$ are given by (1.24). The form of system (1.23) follows from (3.2), (3.4) and (3.8). The proof is complete.

\section{Proof of TheOREM 1.3}

The identity $D J_{3}=J_{3}$ implies

$$
-\frac{f_{x}+f_{u} u_{x}+f_{v} v_{x}+f_{u_{1}} f+f_{v_{1}} g+f_{u_{x}} u_{x x}+f_{v_{x}} v_{x x}}{f}+\frac{2 v_{1} f+u_{1} g}{u_{1} v_{1}+d_{1}}=-\frac{u_{x x}}{u_{x}}+\frac{2 v u_{x}+u v_{x}}{u v+d} .
$$

Comparing the coefficients at $u_{x x}$ and $v_{x x}$ in the above identity, we get

$$
f_{v_{x}}=0, \quad \frac{f_{u_{x}}}{f}=\frac{1}{u_{x}} .
$$

Hence,

$$
f=A\left(x, n, u, v, u_{1}, v_{1}\right) u_{x} .
$$

It follows from the identity $D I_{3}=I_{3}$ that

$$
\frac{f^{\beta_{1}} g}{\left(u_{1} v_{1}+d_{1}\right)^{\beta_{1}}}+\frac{\beta_{1} v_{1}^{2} f^{\beta_{1}+1}}{\left(u_{1} v_{1}+d_{1}\right)^{\beta_{1}+1}}=\frac{u_{x}^{\beta} v_{x}}{(u v+d)^{\beta}}+\frac{\beta v^{2} u_{x}^{\beta+1}}{(u v+d)^{\beta+1}} .
$$


First we consider the case $\beta_{1} \neq \beta$. We have:

$$
g=T v_{x} u_{x}^{\beta-\beta_{1}}+M u_{x}^{1+\beta-\beta_{1}}+N u_{x}
$$

where

$$
T=\frac{A^{-\beta_{1}}\left(u_{1} v_{1}+d_{1}\right)^{\beta_{1}}}{(u v+d)}, \quad M=\frac{\beta v^{2} A^{-\beta_{1}}\left(u_{1} v_{1}+d_{1}\right)^{\beta_{1}}}{(u v+d)^{\beta+1}}, \quad N=-\frac{\beta_{1} v_{1}^{2} A}{\left(u_{1} v_{1}+d_{1}\right)} .
$$

We substitute this expression for $g$ into equation (4.1) and compare the coefficients at $u_{x}^{0}, u_{x}$, $u_{x}^{\beta-\beta_{1}}, v_{x}, u_{x}^{1+\beta-\beta_{1}}$ in the resulting equation. This gives:

$$
\begin{aligned}
& \frac{A_{x}}{A}=0, \\
& \frac{A_{u}}{A}+A_{u_{1}}+\frac{A_{v_{1}} N}{A}+\frac{2 A v_{1}}{u_{1} v_{1}+d_{1}}+\frac{u_{1} N}{u_{1} v_{1}+d_{1}}=\frac{2 v}{u v+d}, \\
& \frac{A_{v}}{A}=\frac{u}{u v+d}, \\
& T\left(\frac{A_{v_{1}}}{A}+\frac{u_{1}}{u_{1} v_{1}+d_{1}}\right)=0, \\
& M\left(\frac{A_{v_{1}}}{A}+\frac{u_{1}}{u_{1} v_{1}+d_{1}}\right)=0 .
\end{aligned}
$$

If $T=0$ or $M=0$, then $A=0$. Hence, in order to have $A \neq 0$, we assume that $T M \neq 0$. If $T M \neq 0$, then equations (4.7)- 4.9 imply

$$
A=\frac{u v+d}{u_{1} v_{1}+d_{a}} S
$$

where $S=S\left(n, u, u_{1}\right)$ is a function depending on $n, u$ and $u_{1}$ only. We substitute the above expression for $A$ into 4.6 and we find that

$$
\left(u_{1} v_{1}+d_{1}\right)^{2}(u v+d) \frac{S_{u}}{S}+\left(u_{1} v_{1}+d_{1}\right)(u v+d)^{2} S_{u_{1}}+v_{1}(u v+d)^{2} S-v\left(u_{1} v_{1}+d_{1}\right)^{2}=0
$$

Then we differentiate the last equation twice with respect to $v_{1}$ and we obtain:

$$
2 u_{1}^{2}(u v+d) \frac{S_{u}}{S}-2 u_{1}^{2} v=0
$$

that is,

$$
\frac{S_{u}}{S}=\frac{v}{u v+d} .
$$

This contradicts to the fact that $S$ is independent of $v, v_{1}$. Hence, $\beta_{1}=\beta$.

We proceed to the case when $\beta$ is a constant, that is, $\beta$ is independent of $n$. Let

$$
\mathcal{D}_{3}=\frac{u_{1} v_{1}+d_{1}}{A(u v+d)}
$$

Then it follows from 4.3 that

$$
g=\left(-\frac{\beta v_{1}^{2}}{\mathcal{D}_{3}(u v+d)}+\frac{\beta v^{2} \mathcal{D}_{3}^{\beta}}{(u v+d)}\right) u_{x}+\mathcal{D}_{3}^{\beta} v_{x} .
$$

Being rewritten in terms of $\mathcal{D}_{3}$, identity 4.1 casts into the form

$$
\begin{aligned}
\frac{\mathcal{D}_{3 x}}{\mathcal{D}_{3}} & +\left(\frac{\mathcal{D}_{3 u}}{\mathcal{D}_{3}}+\frac{u_{1} v_{1}+d_{1}}{\mathcal{D}_{3}^{2}(u v+d)} \mathcal{D}_{3 u_{1}}+\frac{\beta\left(v^{2} \mathcal{D}_{3}^{\beta}-v_{1}^{2} \mathcal{D}_{3}^{-1}\right)}{\mathcal{D}_{3}(u v+d)} \mathcal{D}_{3 v_{1}}+\frac{v_{1}}{\mathcal{D}_{3}(u v+d)}-\frac{v}{(u v+d)}\right) u_{x} \\
& +\left(\frac{\mathcal{D}_{3 v}}{\mathcal{D}_{3}}+\mathcal{D}_{3}^{\beta-1} \mathcal{D}_{3 v_{1}}\right) v_{x}=0 .
\end{aligned}
$$


We compare the coefficients at $u_{x}, v_{x}$ and the free term and we get:

$$
\begin{aligned}
& \mathcal{D}_{3 x}=0 \\
& \frac{u v+d}{\mathcal{D}_{3}} \mathcal{D}_{3 u}+\frac{u_{1} v_{1}+d_{1}}{\mathcal{D}_{3}{ }^{2}} \mathcal{D}_{3 u_{1}}+\frac{\beta v^{2} \mathcal{D}_{3}^{\beta}-\beta v_{1}^{2} \mathcal{D}_{3}^{-1}}{\mathcal{D}_{3}} \mathcal{D}_{3 v_{1}}+\frac{v_{1}}{\mathcal{D}_{3}}-v=0, \\
& \mathcal{D}_{3 v}+\mathcal{D}_{3}^{\beta} \mathcal{D}_{3 v_{1}}=0
\end{aligned}
$$

We introduce a function $W$ in the same way as in the proof of Theorem 1.1 and in new variables

$$
\tilde{v}_{1}=v_{1}-v \mathcal{D}_{3}^{\beta}, \quad \tilde{v}=v, \quad \tilde{u}=u, \quad \tilde{u}_{1}=u_{1}, \quad \tilde{\mathcal{D}}_{3}=\mathcal{D}_{3},
$$

equations 4.14 and 4.13 for the function $W=W\left(n, \tilde{u}, \tilde{v}, \tilde{u}_{1}, \tilde{v}_{1}, \tilde{\mathcal{D}}_{3}\right)$ can be rewritten as follows

$$
\begin{aligned}
& W_{\tilde{v}}=0, \\
& \begin{aligned}
\tilde{\mathcal{D}}_{3}(\tilde{u} \tilde{v}+d) W_{\tilde{u}} & +\left(\tilde{u}_{1}\left(\tilde{v}_{1}+\tilde{v} \tilde{\mathcal{D}}_{3}^{\beta}\right)+d_{1}\right) W_{\tilde{u}_{1}} \\
& +\tilde{\mathcal{D}}_{3}\left(\tilde{v}\left(\tilde{\mathcal{D}}_{3}-\tilde{\mathcal{D}}_{3}^{\beta}\right)-\tilde{v}_{1}\right) W_{\tilde{\mathcal{D}}_{3}}-\beta \tilde{v}_{1}\left(\tilde{v}_{1}+\tilde{v} \tilde{\mathcal{D}}_{3}^{\beta}\right) W_{\tilde{v}_{1}}=0 .
\end{aligned}
\end{aligned}
$$

We differentiate the latter equation with respect to $\tilde{v}$, employ the identity $W_{\tilde{v}}=0$, and get a new system of equations:

$$
\begin{aligned}
& \tilde{u} \tilde{\mathcal{D}}_{3} W_{\tilde{u}}+\tilde{u}_{1} \tilde{\mathcal{D}}_{3}^{\beta} W_{\tilde{u}_{1}}+\left(\tilde{\mathcal{D}}_{3}^{2}-\tilde{\mathcal{D}}_{3}^{\beta+1}\right) W_{\tilde{\mathcal{D}}_{3}}-\beta \tilde{v}_{1} \tilde{\mathcal{D}}_{3}^{\beta} W_{\tilde{v}_{1}}=0, \\
& d \tilde{\mathcal{D}}_{3} W_{\tilde{u}}+\left(\tilde{u}_{1} \tilde{v}_{1}+d_{1}\right) W_{\tilde{u}_{1}}-\tilde{\mathcal{D}}_{3} \tilde{v}_{1} W_{\tilde{\mathcal{D}}_{3}}-\beta \tilde{v}_{1}^{2} W_{\tilde{v}_{1}}=0,
\end{aligned}
$$

which can be rewritten as

$$
\begin{aligned}
& W_{\tilde{u}}+\frac{d_{1} \tilde{\mathcal{D}}_{3}-d_{1} \tilde{\mathcal{D}}_{3}^{\beta}+\tilde{\mathcal{D}}_{3} \tilde{u}_{1} \tilde{v}_{1}}{d_{1} \tilde{u}-d \tilde{\mathcal{D}}_{3}^{\beta} \tilde{u}_{1}+\tilde{u} \tilde{u}_{1} \tilde{v}_{1}} W_{\tilde{\mathcal{D}}_{3}}-\frac{\beta d_{1} \tilde{v}_{1} \tilde{\mathcal{D}}_{3}^{\beta-1}}{d_{1} \tilde{u}-d \tilde{\mathcal{D}}_{3}^{\beta} \tilde{u}_{1}+\tilde{u} \tilde{u}_{1} \tilde{v}_{1}} W_{\tilde{v}_{1}}=0, \\
& W_{\tilde{u}_{1}}-\frac{\tilde{\mathcal{D}}_{3}\left(d \tilde{\mathcal{D}}_{3}-d \tilde{\mathcal{D}}_{3}^{\beta}+\tilde{u} \tilde{v}_{1}\right)}{d_{1} \tilde{u}-d \tilde{\mathcal{D}}_{3}^{\beta} \tilde{u}_{1}+\tilde{u} \tilde{u}_{1} \tilde{v}_{1}} W_{\tilde{\mathcal{D}}_{3}}+\frac{\beta \tilde{v}_{1}\left(d \tilde{\mathcal{D}}_{3}^{\beta}-\tilde{u} \tilde{v}_{1}\right)}{d_{1} \tilde{u}-d \tilde{\mathcal{D}}_{3}^{\beta} \tilde{u}_{1}+\tilde{u} \tilde{u}_{1} \tilde{v}_{1}} W_{\tilde{v}_{1}}=0 .
\end{aligned}
$$

In these equations, we make the change of variables

$$
\begin{aligned}
& u^{*}=\tilde{u} \tilde{v}_{1}^{1 / \beta} d_{1}^{1 /(1-\beta)} \tilde{\mathcal{D}}_{3}^{-1}-d d_{1}^{\beta /(1-\beta)} \tilde{u}_{1} \tilde{v}_{1}^{1 / \beta}, \\
& \mathcal{D}_{3}^{*}=\tilde{v}_{1}^{(1-\beta) / \beta} \tilde{\mathcal{D}}_{3}^{\beta-1}-\tilde{v}_{1}^{(1-\beta) / \beta}+(\beta-1) d_{1}^{-1} \tilde{u}_{1} \tilde{v}_{1}^{1 / \beta}, \\
& u_{1}^{*}=\tilde{u}_{1}, \quad v^{*}=\tilde{v}, \quad v_{1}^{*}=\tilde{v}_{1},
\end{aligned}
$$

and these equations become $W_{v_{1}^{*}}=0$ and $W_{u_{1}^{*}}=0$, respectively. We rewrite these first integrals in old variables and get that the general solution is given implicitly by $H\left(n, K_{3}, L_{3}\right)=0$, where, for each $n$, the symbol $H$ denotes an arbitrary smooth function and $K_{3}, L_{3}$ are given by (1.27), (1.28). The form of system (1.26) follows from (4.2), 4.12) and 4.11). The proof is complete.

\section{ACKNOWLEDGMENTS}

We thank the referee for valuable suggestions and remarks.

\section{REFERENCES}

1. G. Darboux. Leçons sur la théorie générale des surfaces et les applications géométriques du calcul infinitésimal. V. 2. Gautier Villas, Paris (1915).

2. A.B. Shabat, R.I. Yamilov. Exponential systems of type I and Cartan matrices // Preprint, Bashkir Branch of Academy of Sciences of USSR, Ufa (1981). (in Russian).

3. A.N. Leznov, V.G. Smirnov, A.B. Shabat. Internal symmetry group and integrability conditions for two-dimensional dynamical systems // Teor. Matem. Fiz. 51:1, 10-21 (1982). [Theor. Math. Phys. 51:1, 322-330 (1982).] 
4. V.V. Sokolov, A.V. Zhiber. On the Darboux integrable hyperbolic equations // Phys. Lett. A. 208:4-6, 303-308 (1995).

5. A.V. Zhiber, V.V. Sokolov, S.Ya. Startsev. Darboux integrable nonlinear hyperbolic equations // Dokl. Akad. Nauk. 343:6, 746-748 (1995). [Dokl. Math. 52:1, 128-130 (1995).]

6. A.V. Zhiber, V.V. Sokolov. Exactly integrable hyperbolic equations of Liouville type // Uspekhi Matem. Nauk. 56:1(337), 63-106 (2001). [Russ. Math. Surv. 56:1(337), 61-101 (2001).]

7. A.V. Zhiber, R.D. Murtazina. On the characteristic Lie algebras for the equations $u_{x y}=f\left(u, u_{x}\right) / /$ J. Math. Sci. 151:4, 3112-3122 (2008).

8. O.S. Kostrigina, A.V. Zhiber. Darboux-integrable two-component nonlinear hyperbolic systems of equations // J. Math. Phys. 52:3, 033503 (2011)

9. R.D. Murtazina. Nonlinear hyperbolic equations with characteristic ring of dimension 3 // Ufimskij Matem. Zhurn. 3:4, 113-118 (2011). (in Russian).

10. I.M. Anderson, M.E. Fels. The Cauchy problem for Darboux integrable systems and non-linear d'Alembert formulas // SIGMA. 9, 017 (2013).

11. I.M. Anderson, M.E. Fels, P.J. Vassiliou. On Darboux integrability // in "Symmetry and perturbation theory", Proc. 6th Int. Conf. Italy, 2007. World Scientific, Hackensack, 13-20 (2008).

12. A.B. Zhiber, R.D. Murtazina, I.T. Habibullin, A.B. Shabat. Characteristic Lie rings and integrable models in mathematical physics // Ufimskij Matem. Zhurn. 4:3, 17-85 (2012). [Ufa Math. J. 4:3, 17-85 (2012). $]$

13. I.T. Habibullin, A. Pekcan. Characteristic Lie algebra and the classification of semi-discrete models // Theor. Math. Phys. 151:3, 781-790 (2007).

14. V.E. Adler, S.Ya. Startsev. On discrete analogues of the Liouville equation// Teor. Matem. Fiz. 121:2, 217-284 (1999). [Theor. Math. Phys. 121:2, 1484-1495 (1999).]

15. I.T. Habibullin. Characteristic algebras of fully discrete hyperbolic type equations // SIGMA. 1, $023(2005)$.

16. I.T. Habibullin, K. Zheltukhin, M. Yangubaeva. Cartan matrices and integrable lattice Toda field equations // J. Phys. A 44:46, 465202 (2011).

17. I.T. Habibullin, N. Zheltukhina, A. Pekcan. On some algebraic properties of semi-discrete hyperbolic type equations // Turkish J. Math. 32:3, 277-292 (2008).

18. I.T. Habibullin, N. Zheltukhina, A. Pekcan. On the classification of Darboux integrable chains // J. Math. Phys. 49:10, 102702 (2008).

19. I.T. Habibullin, N. Zheltukhina, A. Pekcan. Complete list of Darboux integrable chains of the form $t_{1 x}=t_{x}+d(t, t 1) / /$ J. Math. Phys. 50:10, 102710 (2009).

20. I.T. Habibullin, N. Zheltukhina, A. Sakieva. On Darboux-integrable semi-discrete chain // J. Phys. A 43:43, 434017 (2010).

21. I. Habibullin, N. Zheltukhina, A. Sakieva. Discretization of hyperbolic type Darboux integrable equations preserving integrability // J. Math. Phys. 52:9, 093507 (2011).

22. I.T. Habibullin, E.V. Gudkova. Classification of integrable discrete Klein-Gordon models // Physica Scripta. 83:4, 045003 (2010).

23. S.V. Smirnov. Semidiscrete Toda lattices // Teor. Matem. Fiz. 172:3, 387-402 (2012). [Theor. Math. Phys. 172:3, 1217-1231 (2012).]

24. I.T. Habibullin, N. Zheltukhina. Discretization of Liouville type nonautonomous equations // J. Nonl. Math. Phys. 23:4, 620-642 (2016).

25. K. Zheltukhin, N. Zheltukhina. On the discretization of Laine equations // J. Nonl. Math. Phys. 25:1, 166-177 (2018).

26. K. Zheltukhin, N. Zheltukhina. On the discretization of Darboux integrable Systems // J. Nonl. Math. Phys. 27:4, 616-632 (2020).

Kostyantyn Zheltukhin,

Department of Mathematics, Middle East Technical University,

Ankara, Turkey

E-mail: zheltukh@metu.edu.tr 
Natalya Zheltukhina,

Department of Mathematics, Faculty of Science, Bilkent University, Ankara, Turkey

E-mail: natalya@fen.bilkent.edu.tr 